\title{
Synthesis of a Hein-Schiff base compound and its antibacterial activity on cotton fabrics
}

\author{
Wen Wen $\cdot$ Zaixing Zhang $\cdot$ Lingxiao Jing $\cdot$ Tonghua Zhang
}

Received: 11 February 2020/ Accepted: 2 April 2020/Published online: 28 May 2020

(C) Springer Nature B.V. 2020

\begin{abstract}
This work aimed to obtain a highly efficient and durable antimicrobial agent on cotton fabrics by synthesizing a new Schiff base compound called (E)-1-((4-(allyloxy) benzyl) amino) imidazolidine-2,4-dione (AOBYID). Cotton fabrics were finished with Schiff base compound via alkali-free thiol-ene click chemistry. Chlorine were subsequently added to the nitrogen-containing groups on the grafted cotton fabrics through chlorination with sodium hypochlorite. NMR, FT-IR, Raman, and SEM results showed that the target compound (AOBYID) was successfully synthesized and effectively processed on
\end{abstract}

cotton fabrics. Antibacterial test results showed that the antibacterial rates of the treated fabrics after $1 \mathrm{~min}$ of contact reached $97.83 \%$ and $94.83 \%$ for $E$. coli and S. aureus, respectively, and remained above $90 \%$ after $24 \mathrm{~h}$ contact. In addition, the treated fabrics could retain approximately $79 \%$ of the initial chlorine content after 30 days storage, and $60 \%$ of the chlorine could be regenerated after $12 \mathrm{~h}$ of UV light. These findings revealed the high efficiency and durability of the antibacterial activity of treated cotton fabrics.
W. Wen · L. Jing · T. Zhang

Chongqing Engineering Research Center of Biomaterial

Fiber and Modem Textile, College of Textiles \&

Garments, Southwest University, Chongqing 400715,

China

\section{Z. Zhang}

Hunan Engineering Laboratory for Preparation

Technology of Polyvinyl Alcohol Fiber, Chemistry and

Material Engineering School of Huaihua University,

Huaihua 418008, China

T. Zhang ( $\square)$

No. 2 Tiansheng Road, Beibei, Chongqing 400716, China

e-mail: zhtonghua@aliyun.com 


\section{Graphic abstract}

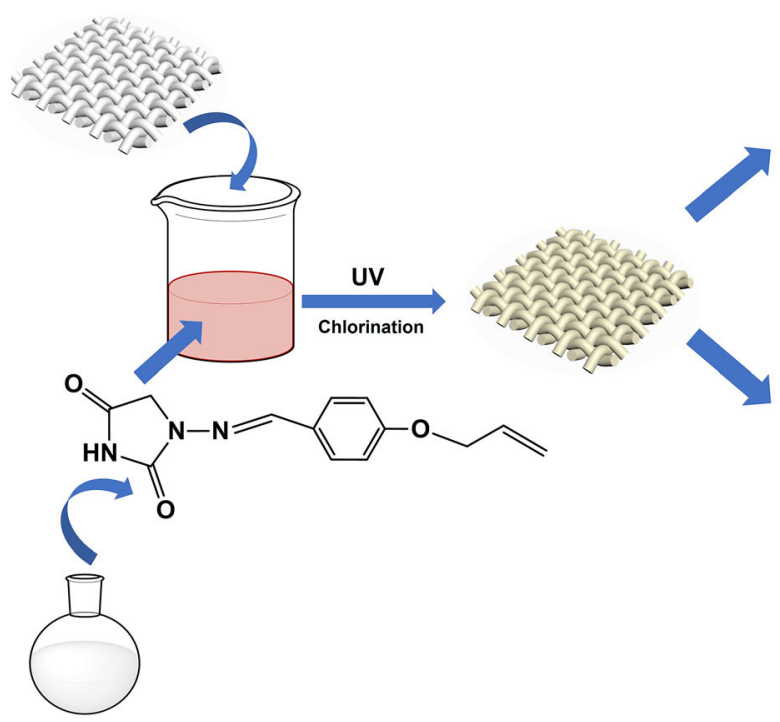

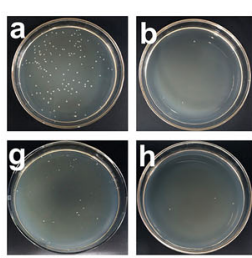

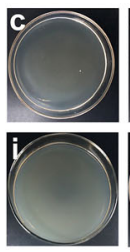

E. coli

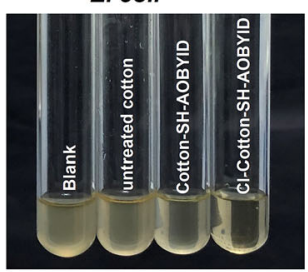

E. coli
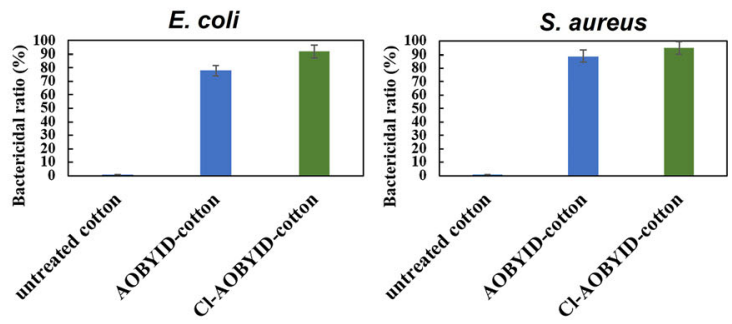

Keywords Schiff base compound - Cotton fabric . Thiol-ene click · Antibacterial · Stability

\section{Introduction}

Cotton is an abundant natural fiber consisting of practically pure cellulose (approximately 88-96\%) (Rattanaphani et al. 2007). Among the natural fibers, cotton is widely used in daily life due to its good hygroscopicity, soft comfort, and biodegradability. However, cotton fabrics propagate the reproduction of microorganisms, such as bacteria and fungi that will spread diseases, remarkably increase cross-infection risk, and even endanger human health (Küçük and Öveçoğlu 2019). Hence, understanding the antibacterial activities of cotton fabrics is crucial.

Various types of antibacterial agents, such as $\mathrm{N}$ halamine (Chen et al. 2020), quaternary ammonium salts (Liu et al. 2019), nanosilver (Rohani Shirvan et al. 2019), and chitosan (Zhang et al. 2018), have been developed to determine antibacterial activity. Among these agents, $N$-halamine has attracted researchers' attention due to its unique structure, nontoxicity, and highly effective antibacterial properties. $\mathrm{N}$-Halamine is a group of compounds containing one or more nitrogen-halogen covalent bonds $(\mathrm{N}-\mathrm{Cl})$ normally formed via the halogenation of imide, amide, or amine groups (Qiao et al. 2017). Moreover, $N$ halamine has antibacterial activity against a broad spectrum of microorganisms, including bacteria, viruses, fungi, and yeasts (Ma et al. 2019a, b).

When the oxidative halogen in N-halamine is consumed by sterilization, it can be restored through the dilution of household bleach or halogen-releasing agents (Ma et al. 2019a, b). However, poor stability of $\mathrm{N}$-Halamine compounds against UV light can limit their practical applications. Ren et al. (2008) confirmed that UV light irradiation could rapidly dissociate the $\mathrm{N}-\mathrm{Cl}$ bonds in siloxane precursors and cause unrecoverable loss of antibacterial activity related to the cleavage between $N$-halamine functional groups and cotton substrates. However, Sandstrom et al. (2007) reported that $N$-halamine compounds with aromatic structures could partially protect the $\mathrm{N}-\mathrm{Cl}$ bonds from breakage. Moreover, the Schiff bases with imino groups $(\mathrm{RC}=\mathrm{N})$ possessing special structures and variable properties have been widely used in biological activities and functional materials (Naeimi 
et al. 2008; Hassan et al. 2018; Yousif et al. 2018). However, most studies were based on the Schiff base complex and only a few used the Schiff base in fabric finishing. Furthermore, the highly efficient introduction of functional molecules on cotton fabric surface by using a facile method continues to be an important research topic.

Metal-free thiol-ene click reactions have attracted researchers' interest because of their unique advantages of simplicity, high efficiency, strong versatility, and mild reaction conditions. Although their applications have been widely studied, such as in compound synthesis, material surface modification, and fabric functional finishing, most works typically focused on the thiol-Michael addition reactions and ignored the antibacterial finishing of cotton fabrics (Liu et al. 2017; Fei et al. 2018; Herwig and Dove 2019). These reactions require the use of nucleophiles, such as strong bases, metals, organometals, and Lewis acids, which hinder environmental protection. By contrast, the thiol-ene click reaction can be initiated by adding even a small amount of photocatalyst, and the reaction is highly efficient.

In this work, a Schiff base compound with Hein, double bond, and benzene ring was synthesized, and an alkali-free thiol-ene click reaction was introduced to the antibacterial finish of cotton fabrics. First, a sulfhydryl group was introduced onto the surface of cotton fibers with a 3-mercaptopropyltriethoxysilane (MPTES) treatment. This group could efficiently react with the terminal olefinic bonds of E-1-((4-(allyloxy) benzyl) amino) imidazolidine-2,4-dione (AOBYID) and form covalent bonds to improve the stability of AOBYID remarkably. Then, chlorine was introduced into the nitrogen-containing groups on the grafted cotton fabric to transform it into a highly effective antibacterial substance via sodium hypochlorite chlorination. The possible reaction between AOBYID and cellulose is presented in Scheme 1. In addition, the antibacterial properties, stability, and rechargeability of the treated samples were explored further.

\section{Experiment}

\section{Materials}

Bleached cotton fabric $\left(144 \mathrm{~g} / \mathrm{m}^{2}\right)$ was used in this study. P-hydroxybenzaldehyde, 3-bromopropene, 1-aminohein hydrochloride, $N, N$-dimethylformamide (DMF), petroleum ether, sodium chloride, and sodium thiosulfate solution $(0.1000 \mathrm{~mol} / \mathrm{L})$ were purchased from Shanghai Aladdin Biochemical Technology Co., Ltd. Sodium hydroxide and ethanol were sourced from Chongqing Chuandong Chemical (Group) Co., Ltd. 3-mercap-topropyltriethoxysilane (MPTES), sodium hypochlorite solution (6-14\% active chlorine basis), and beef paste were obtained from Shanghai Macklin Biochemical Technology Co., Ltd. Peptone. Benzoin dimethyl ether (DMPA) was supplied by Shanghai Yuanye Biological Technology Co., Ltd. Agar powder was acquired from Chengdu Kelong Chemical Co., Ltd. AOBYID was synthesized using 3-bromopropene, P-hydroxybenzaldehyde, and 1-aminohein hydrochloride. All chemicals were analytic grade reagents and used without further purification.

\section{AOBYID synthesis}

The synthesis process of AOBYID is shown in Scheme 2. First, sodium hydroxide (4 g, $0.1 \mathrm{~mol}$ ) and P-hydroxybenzaldehyde (12.212 g, $0.1 \mathrm{~mol})$ were initially added to a round bottom flask $(250 \mathrm{~mL})$ containing $100 \mathrm{ml}$ ethanol and stirred at room temperature until dissolved. Second, 3-bromopropene $(12.098 \mathrm{~g}, 0.1 \mathrm{~mol})$ was added dropwise thereto, placed in an oil bath with a magnetic stirrer, and stirred under reflux at $70{ }^{\circ} \mathrm{C}$ for $16 \mathrm{~h}$. Third, the salt was filtered under reduced pressure, the solvent ethanol was evaporated, and the residue was purified with petroleum ether to obtain a pale-yellow liquid (4(allyloxy)benzaldehyde). Fourth, 1-aminohydantoin hydrochloride $(15.155 \mathrm{~g}, 0.1 \mathrm{~mol})$ was added to a round-bottomed flask $(250 \mathrm{~mL})$ containing $60 \mathrm{~mL}$ ethanol, 4-(allyloxy) benzaldehyde $\quad(17.700 \mathrm{~g}$, $0.1 \mathrm{~mol})$ was poured into simultaneously, and then stirred under reflux at $70{ }^{\circ} \mathrm{C}$ for $18 \mathrm{~h}$. After the reaction, the solvent was evaporated under reduced pressure, and deionized water was used to precipitate a solid to obtain a crude product. Finally, a light yellowsolid powder (AOBYID) was obtained with recrystallization in petroleum ether.

\section{Modification of cotton fabric with MPTES}

First, the cotton fabric was cleaned with an aqueous solution at $80{ }^{\circ} \mathrm{C}$ for $20 \mathrm{~min}$ to remove the surface impurities and then dried at $60{ }^{\circ} \mathrm{C}$. Second, anhydrous 

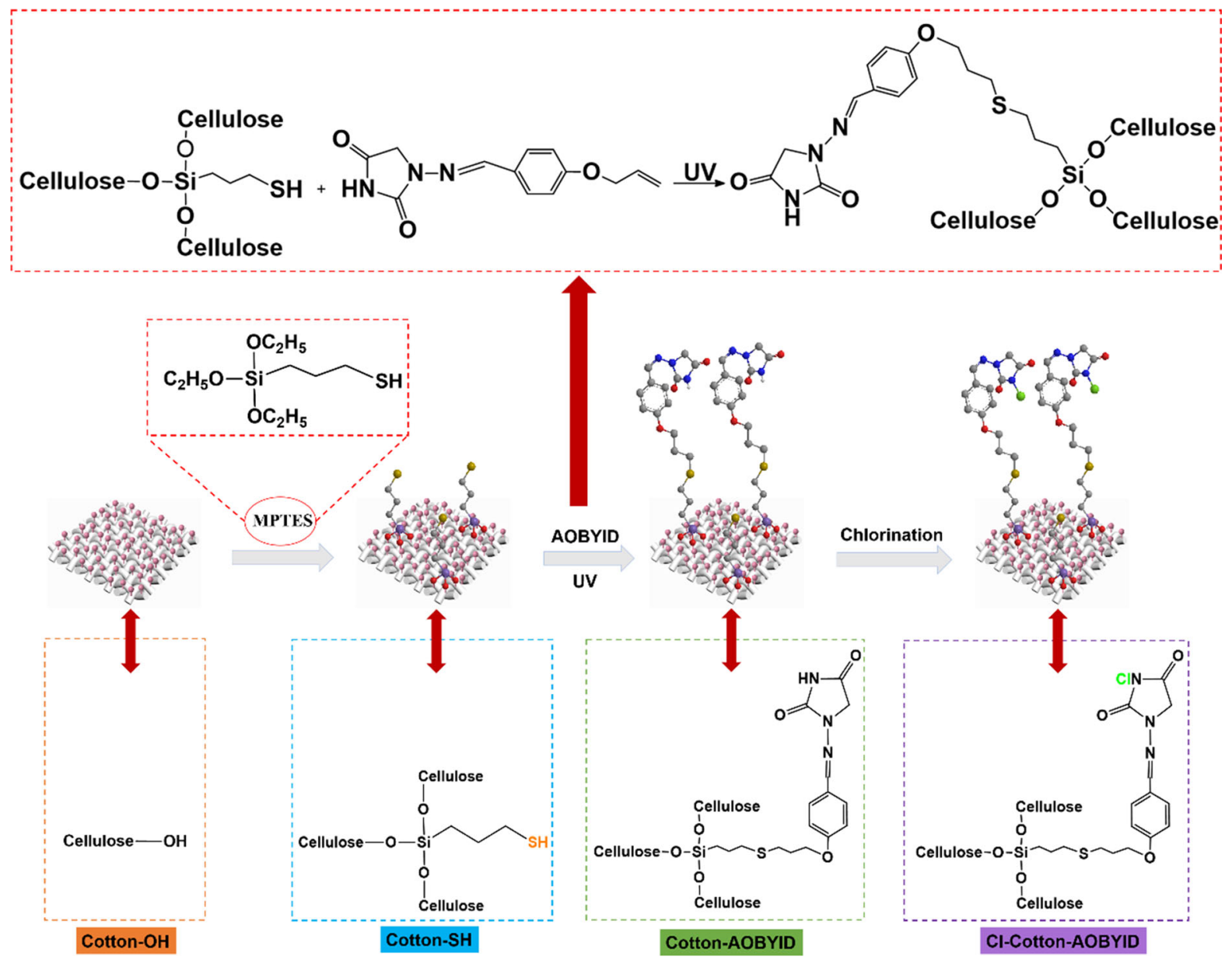

Scheme 1 Mechanism of preparing antibacterial cotton fabric

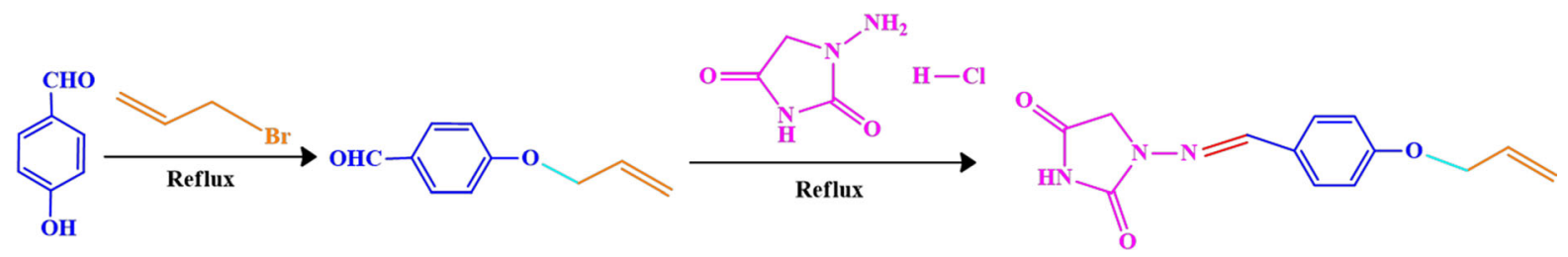

Scheme 2 Preparation procedure of AOBYID

ethanol and distilled water in the ratio of $4: 1$ was mixed in the solution, and MPTES (5 wt $\%$ ) was added. Third, the $\mathrm{pH}$ of the mixture was adjusted to 3.5 with acetic acid. Fourth, the mixed solution was continuously stirred at $25{ }^{\circ} \mathrm{C}$ for $30 \mathrm{~min}$, and the cotton fabric was immersed into the above solution for $3 \mathrm{~min}$ and solidified at $25^{\circ} \mathrm{C}$ for $5 \mathrm{~min}$. Finally, the MPTESmodified cotton (cotton-SH) was obtained.
Finishing of cotton fabric via the addition of alkalifree thiol-ene click chemistry

First, the target compound was dissolved in DMF, and then $0.1 \%$ wt DMPA was added as a photoinitiator with a bath ratio of 1:20. Second, cotton-SH was dipped into the mixture solution and treated with UV light for $30 \mathrm{~min}$, and AOBYID was grafted onto the cotton fabrics in the form of $\mathrm{C}-\mathrm{S}$ bonds through the 
thiol-ene click reaction. Third, the fabrics were washed thoroughly and dried at $45^{\circ} \mathrm{C}$ to obtain the AOBYID-grafted cotton (cotton-SH-AOBYID).

Grafting rate of the cotton fabrics

The average grafting rate percentage $(\mathrm{G} \%)$ of each sample was calculated as follows:

$\mathrm{G} \%=\frac{M_{2}-M_{1}}{M_{1}} \times 100 \%$

where $M_{1}$ and $M_{2}$ are the weights of the sample before and after the MPTES grafting treatment, respectively.

All the samples were placed in $60 \%$ relative humidity at $25{ }^{\circ} \mathrm{C}$ for $24 \mathrm{~h}$ before measurement. According to Eq. (1), the graft ratio of antibacterial cotton fabric was calculated at $15.7 \%$, which was obtained by averaging the results after five measurements.

Chlorination and determination of chlorine loading

To obtain the antibacterial cellulose of N-halamine Schiff base and render the fabrics with high biocidal efficacy, the treated cotton fabric was soaked in $10 \%$ sodium hypochlorite solution $(\mathrm{pH} \mathrm{7})$ for $1 \mathrm{~h}$, and then washed with deionized water and dried at $45{ }^{\circ} \mathrm{C}$ for $1 \mathrm{~h}$. The chlorine loading on the fabrics was measured using the iodometric/thiosulfate titration method. The amount of oxidative chlorine on the cotton swatches was calculated as follows:

$\mathrm{Cl}^{+} \%=\frac{\mathrm{N} \times \mathrm{V} \times 35.45}{\mathrm{~W} \times 2} \times 100 \%$

where $\mathrm{Cl}^{+} \%$ is the wt.\% of oxidative chlorine in the samples; $\mathrm{N}$ and $\mathrm{V}$ are the normality (equiv./L) and volume (L) of titrant sodium thiosulfate, respectively; and $\mathrm{W}$ is the weight of the cotton samples $(\mathrm{g})$.

\section{Characterization}

Nuclear magnetic resonance $\left({ }^{1} \mathrm{H}\right.$ NMR)

Nuclear magnetic resonance $\left({ }^{1} \mathrm{H}\right.$ NMR) spectra of AOBYID are obtained via a WNMR-I-400 MHz with TMS as the internal standard using DMSO.
Fourier transform-infrared spectroscopy (FT-IR)

The infrared spectra of AOBYID, untreated cotton, cotton-SH, and cotton-SH-AOBYID were obtained using a GX infrared spectrometer (PE Co., USA) under the following test conditions: the ratio of sample to $\mathrm{KBr}$ of $1: 100$, wavenumber range of $4000-500 \mathrm{~cm}^{-1}$, number of scans of 32 , and resolution of $4 \mathrm{~cm}^{-1}$.

Raman analysis

The surface functional groups after the MPTES pretreatment were investigated with a spectral range of $2000-3000 \mathrm{~cm}^{-1}$ by using the Raman analysis (DXR2, ThermoFisher Scientific, USA).

Scanning electron microscopy (SEM)

The surface morphology images of untreated cotton, cotton-SH, and cotton-SH-AOBYID, and chlorinated (Cl-cotton-SH-AOBYID) samples were observed using SEM (Hitachi S-4800, the Netherlands) at an accelerating voltage of $10 \mathrm{kV}$.

Antibacterial activity test

The antibacterial activities of Cl-cotton-SH-AOBYID were evaluated through the zone of inhibition, number of colony-forming units (CFU), and optical density at $600 \mathrm{~nm}$. The control and chlorinated cotton swatches were challenged with Staphylococcus aureus ( $S$. aureus, gram-positive organism) and Escherichia coli (E. coli, gram-negative organism).

In the CFU counting method, $25 \mu \mathrm{L}$ aliquot of bacterial suspension $(100 \mathrm{mM}$ phosphate buffer, $\mathrm{pH}=$ 7) was added to the center of two $2.54 \mathrm{~cm}^{2}$ cotton swatches, which were held in place with sterile weights to ensure good contact of the swatches with the inoculum. The number of bacteria is used in the tests for approximately $10^{6} \mathrm{CFUs}(\mathrm{CFU} / \mathrm{mL})$, and the actual bacterial count was determined through the counting method. After the contact times of 1, 5, 10, and $30 \mathrm{~min}$, the samples were transferred immediately to conical tubes containing $5 \mathrm{~mL}$ sterile $0.02 \mathrm{~N}$ sodium thiosulfate solution and vortexed for $2 \mathrm{~min}$ to remove all oxidative chlorine and rinse off any surviving bacteria. The serial dilutions of the quenched solution samples were made using $100 \mu \mathrm{M}$ 
phosphate buffer $(\mathrm{pH}$ 7) and plated on a LuriaBertani medium. After incubation at $37^{\circ} \mathrm{C}$ for $24 \mathrm{~h}$, the viable bacterial colonies were recorded for biocidal efficacy analysis. The same procedure was performed on the untreated asepsis cotton as control but with only $30 \mathrm{~min}$ of contact. The antibacterial rate (R, \%) was calculated using Eq. (3) (Ma et al. 2013; Rong et al. 2019).

$\mathrm{R}(\%)=\frac{\mathrm{CFU} \text { in control }-\mathrm{CFU} \text { in sample }}{\mathrm{CFU} \text { in control }} \times 100 \%$

In the inhibition zone method, LB agar medium was prepared and autoclaved at $121{ }^{\circ} \mathrm{C}$ for $20 \mathrm{~min}$ of sterilization before use. The liquid LB agar medium was then cooled to $45^{\circ} \mathrm{C}$ and mixed with an $E$. coli or S. aureus broth to a concentration of $10^{6} \mathrm{CFU} / \mathrm{mL}$ and poured on dishes. Each sample was subsequently placed on the dish, which were incubated at $37{ }^{\circ} \mathrm{C}$ for $24 \mathrm{~h}$ before observation. The zones of inhibition were recorded with a camera (Cai et al. 2018).

In the OD600 measurement, the antibacterial activity of the three sample groups, including untreated cotton, AOBYID-SH-cotton, and Cl-cotton-SH-AOBYID samples, were tested by evaluating the inhibition of the bacteria $S$. aureus and E. coli. Then, $100 \mu \mathrm{L}$ bacterial suspension $\left(10^{6} \mathrm{CFU} \mathrm{mL}^{-\mathbf{1}}\right)$ was added to the samples. After $4 \mathrm{~h}, 900 \mu \mathrm{L}$ of the Luria-Bertani broth was added. The samples were subsequently placed in an incubator at $37^{\circ} \mathrm{C}$ with constant agitation. After $24 \mathrm{~h}, 200 \mu \mathrm{L}$ of bacterial suspension was collected. The optical density (OD) of the suspension at $600 \mathrm{~nm}$ was measured using a microplate reader. The bactericidal ratios of the groups were calculated using Eq. (4) (Gan et al. 2019).

$$
\begin{aligned}
& \text { Bactericidal ratio }(\%)= \\
& \frac{\text { OD of contrastive group }- \text { OD of experimental group }}{\text { OD of contrastive group }} \\
& \times 100 \%
\end{aligned}
$$

\section{Storage stability testing}

In the storage stability test, the chlorinated cotton fabrics were stored in the dark at room temperature, and the chlorine loadings were measured at different periods of times. The samples were rechlorinated and the chlorine loadings were retitrated.

UV light stability testing

The chlorinated cotton fabrics were placed in a UV light chamber for the duration of 1 to $12 \mathrm{~h}$. After the specified time of UV irradiation, the cotton swatches were removed from the UV chamber and titrated or rechlorinated and titrated according to the previously described method.

\section{Results and discussion}

\section{Characterization of AOBYID}

The FT-IR and ${ }^{1} \mathrm{H}$ NMR spectra were used to investigate the synthesis of the target compound of the Hein-Schiff base. The characterizations of the synthesized AOBYID were first carried out via ${ }^{1} \mathrm{H}$ NMR spectroscopy (Fig. 1a). The following results were obtained. ${ }^{1} \mathrm{H}$ NMR (DMSO) $\delta_{\mathrm{H}}: 11.15(\mathrm{~s}, 1 \mathrm{H}$, $\mathrm{HN})(\mathrm{a} 1), 7.75(\mathrm{~s}, 1 \mathrm{H}, \mathrm{HC}-10)(\mathrm{a} 2), 7.63$ (d, $\mathrm{J}=8.68 \mathrm{~Hz}, 1 \mathrm{H}, \mathrm{HC}-2)(\mathrm{a} 3), 7.63$ (d, J = $8.68 \mathrm{~Hz}$, 1H, HC-6) (a3), 7.02 (d, J = $8.88 \mathrm{~Hz}, 1 \mathrm{H}, \mathrm{HC}-3)(\mathrm{a} 4)$, $7.02(\mathrm{~d}, \mathrm{~J}=8.88 \mathrm{~Hz}, 1 \mathrm{H}, \mathrm{HC}-5)(\mathrm{a} 4), 6.05(\mathrm{~m}$, $\mathrm{J}=27.84 \mathrm{~Hz}, \quad 1 \mathrm{H}, \quad \mathrm{HC}-8) \quad(\mathrm{a} 5), \quad 5.41 \quad$ (dd, $\mathrm{J}=17.3 \mathrm{~Hz}, 1 \mathrm{H}, \mathrm{HC}-9)(\mathrm{a} 6), 5.27$ (q, J = $11.8 \mathrm{~Hz}$, 1H, HC-9) (a7), 4.61 (d, J = 5.12 Hz, 1H, HC-7) (a8), and 4.33 (s, 2H, HC-13) (a9). These signals indicated that the Hein-Schiff base compound might have been successfully synthesized.

The structure of the target compound was further verified through its FT-IR spectra (Fig. 1b). The two peaks around 3196 and $3075 \mathrm{~cm}^{-1}$ are due to the $\mathrm{N}-\mathrm{H}$ stretching vibration in hydantoin. The weak peaks at 693 and $618 \mathrm{~cm}^{-1}$ correspond to the out-of-plane rocking vibration of $\mathrm{N}-\mathrm{H}$ and $\mathrm{O}=\mathrm{C}-\mathrm{N}$ in hydantoin, respectively. Furthermore, the absorption peak in the $1790-1765 \mathrm{~cm}^{-1}$ region was generally ascribed to the symmetrically coupled vibration of $\mathrm{C}=\mathrm{O}$ in hydantoin, whereas the signal at the $1755-1690 \mathrm{~cm}^{-1}$ region was related to the asymmetrically coupled vibration of $\mathrm{C}=\mathrm{O}$. The peaks appearing at 1513 and $1248 \mathrm{~cm}^{-1}$ belong to the bending vibration of $\mathrm{C}-\mathrm{N}-\mathrm{H}$ and the 
a
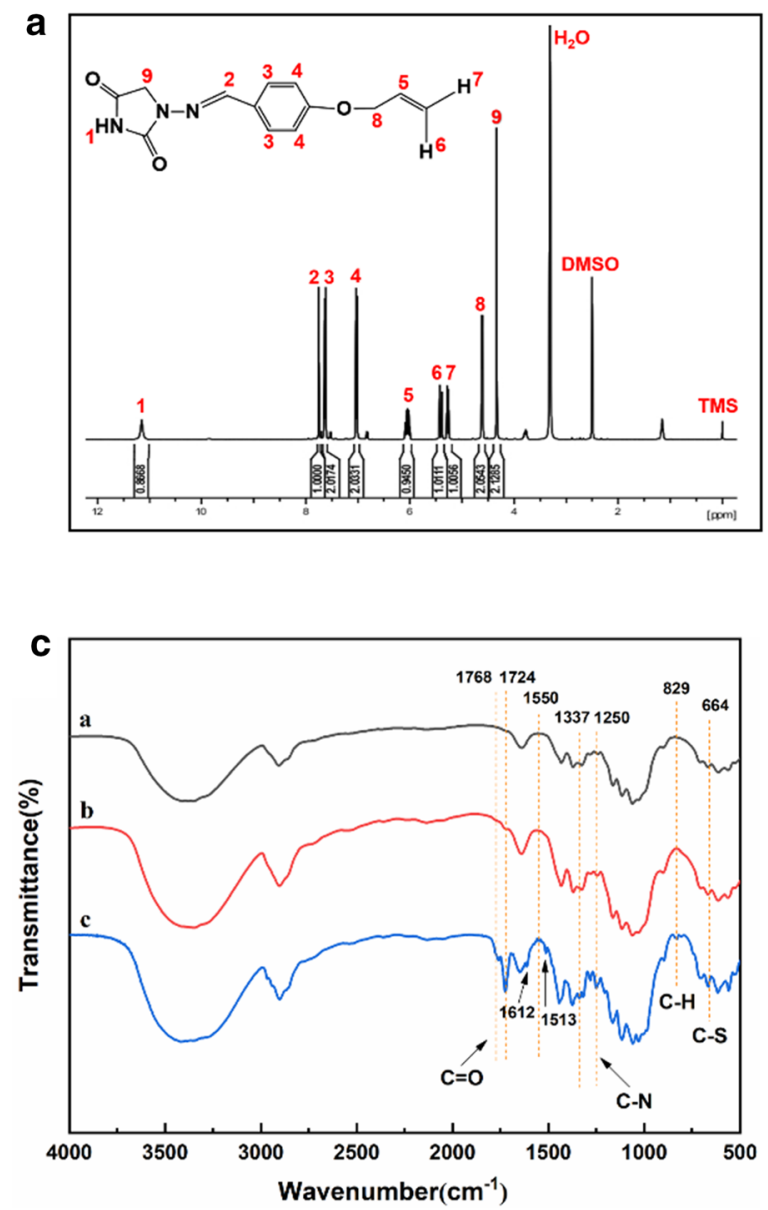
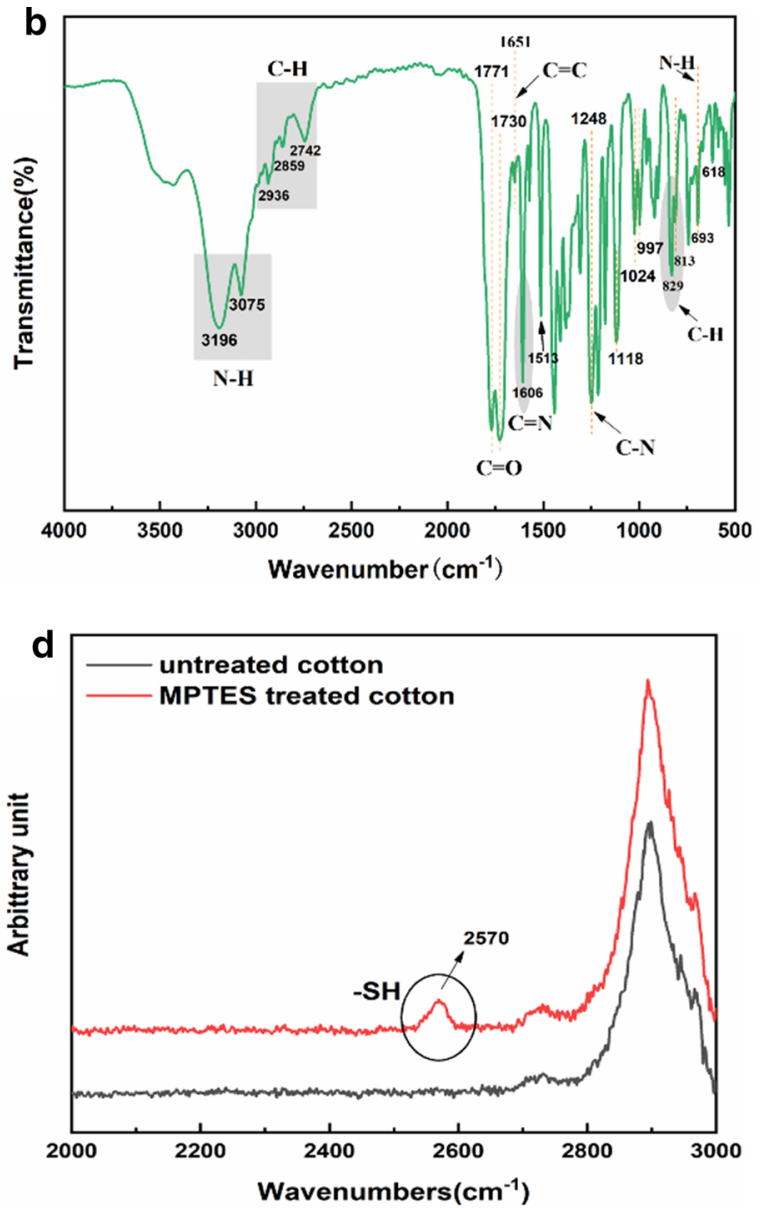

Fig. 1 a ${ }^{1} \mathrm{H}$ NMR spectrum, and b FT-IR spectra of AOBYID; c FT-IR spectra of a: untreated cotton, b: cotton-SH, and c: cotton-SHAOBYID; d Raman spectra images of untreated cotton and cotton-SH

telescopic vibration of $\mathrm{C}-\mathrm{N}$ in hydantoin, respectively. The weak peaks at 2936,2859 , and $2742 \mathrm{~cm}^{-1}$ were assigned to the $\mathrm{C}-\mathrm{H}$ stretching vibration in the benzene ring. The absorption peaks in the 1500-990 and $860-790 \mathrm{~cm}^{-1}$ regions were generally attributed to the in-plane bending vibration and out-of-plane bending vibration of $\mathrm{C}-\mathrm{H}$ in the benzene ring. In addition, the peak in the $1700-1610 \mathrm{~cm}^{-1}$ region was usually ascribed to the $\mathrm{C}=\mathrm{C}$ telescopic vibration. Therefore, the absorption band detected at $1651 \mathrm{~cm}^{-1}$ should belong to the terminal alkenyl group of AOBYID. Meanwhile, the peak appearing at $1606 \mathrm{~cm}^{-1}$ belongs to the characteristic active group $\mathrm{C}=\mathrm{N}$ of the Schiff base. These results are in accordance with the ${ }^{1} \mathrm{H}$ NMR spectroscopy analysis.
Therefore, the Hein-Schiff base compound was successfully synthesized.

FT-IR and Raman spectroscopy analysis

Figure 1c shows the FT-IR spectra of untreated cotton, cotton-SH, and cotton-SH-AOBYID. Compared with the untreated cotton sample, cotton-SH essentially remained unchanged. However, two characteristic absorption peaks were observed near 1768 and $1724 \mathrm{~cm}^{-1}$ associated with the stretching vibration of $\mathrm{C}=\mathrm{O}$ groups of AOBYID molecules in the curve of the treated cotton. The skeleton vibration peaks of phenyl ring appeared at 1612, 1550, and $1513 \mathrm{~cm}^{-1}$. The two peaks observed around 1337 and $1250 \mathrm{~cm}^{-1}$ in the cotton-SH-AOBYID fabrics 
indicated that a characteristic absorption peak of the $\mathrm{C}-\mathrm{N}$ bond occurred in imide ( $\mathrm{Xu}$ et al. 2017). The absorption peak at $829 \mathrm{~cm}^{-1}$ demonstrated the $\mathrm{C}-\mathrm{H}$ stretching vibration of phenyl in the cotton-SHAOBYID fabrics. The peak at $664 \mathrm{~cm}^{-1}$ exhibited the vibration absorption peak of the $\mathrm{C}-\mathrm{S}$ bond, which was generated by the reaction of AOBYID with -SH. Based on these analyses, a reaction occurred between AOBYID and a thiol group through click chemistry.

The molecular identities of MPTES on the cotton fabrics were assessed through their Raman spectra. As shown in Fig. 1d, the characteristic signal around $2570 \mathrm{~cm}^{-1}$ could be found in the curves of the treated cotton fabric due to the -SH vibration band (Xu et al. 2017). This result proved that hydroxyl in the cotton fabric reacted with MPTES to generate the -SH group, which was ready for the thiol-ene click reaction in the following step.
Surface morphology

SEM tests were carried out to observe the surface morphological characteristics of the samples. As shown in Fig. 2a, the original surface of cotton fibers presented a typical morphology of the natural structures of grain and ravines, which are slightly rough but relatively smooth with irregularly shaped fibers (Fan et al. 2019). After the MPTES treatment, a slight change occurred on its surface, and the change is unclear (Fig. 2b). Furthermore, Fig. 2c also shows that the surface of the AOBYID-treated cotton fibers appear to have a thin coating, thereby suggesting the occurrence of grafting of the AOBYID compound onto the MPTES-modified cotton fabrics. After chlorination (Fig. 2d), the cotton fibers became slightly rough and a layer of ribbon-like material covered the surface of the original cotton fibers, thereby
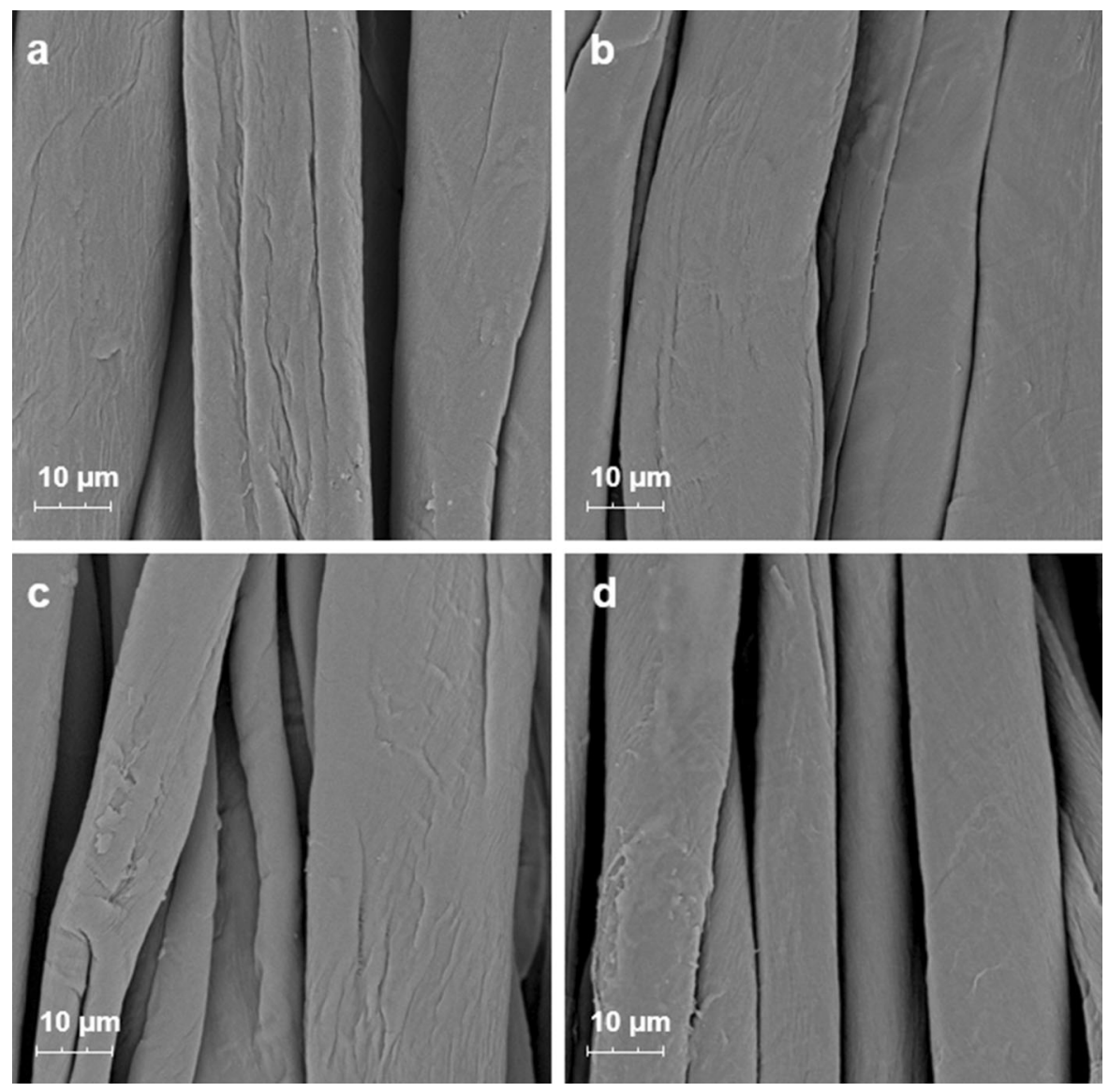

Fig. 2 SEM images of the a untreated cotton, b cotton-SH, c cotton-SH-AOBYID, and d Cl-cotton-SH-AOBYID fabrics 
suggesting that the AOBYID-grafted cotton was successfully chlorinated.

\section{Antibacterial efficacies}

Figure 3 presents the results of E. coli and $S$. aureus living on the Petri dishes when the sample is in contact with the inoculum at a concentration of $10^{6} \mathrm{CFU} / \mathrm{mL}$ at different times.

The original data are listed in Table 1. Figure 3a-1 illustrate the results of the antibacterial properties against $E$. coli and $S$. aureus, respectively. As shown in Fig. 3f and 1, compared with the untreated cottonadded antibiotics, untreated cotton and Cl-cotton-SHAOBYID did not produce zones of inhibition against E. coli or $S$. aureus. The results revealed that the
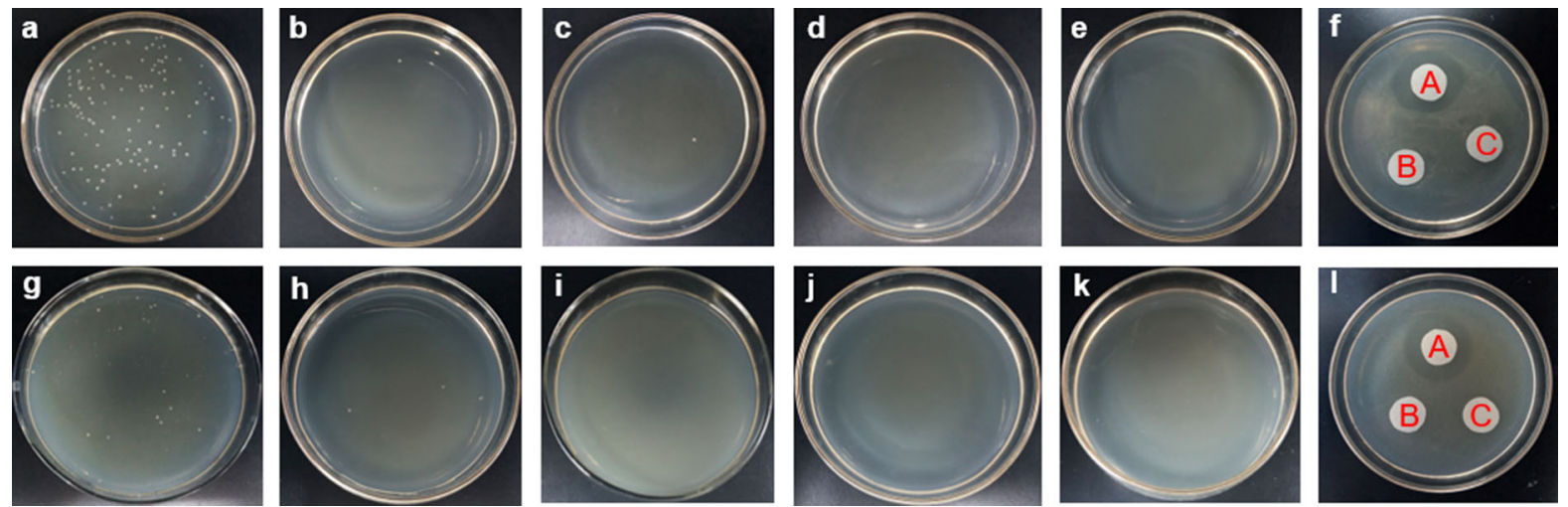

Fig. 3 Antibacterial properties of samples against E. coli: a untreated cotton with contact of $30 \mathrm{~min}$, b-e Cl-cotton-SHAOBYID with contact of $1,5,10$, and $30 \mathrm{~min}$. Antibacterial properties of samples against $S$. aureus: $\mathbf{g}$ untreated cotton with contact of $30 \mathrm{~min}, \mathbf{h}-\mathbf{k}$ Cl-cotton-SH-AOBYID with contact of antibacterial abilities of Cl-cotton-SH-AOBYID were investigated through the CFU counting method, which is an effective quantitative antibacterial test that analyzes the inhibitory activity.

The bacteriostatic rates of Cl-cotton-SH-AOBYID against $E$. coli and $S$. aureus reached $97.83 \%$ and $94.83 \%$, respectively, after a contact period of only 1 min (Fig. 3b, h). Notably, the antibacterial efficiency was above $99 \%$ (Fig. 3c, i) after 5 min of contact and achieved $100 \%$ against S. aureus. This finding confirmed that Cl-cotton-SH-AOBYID obtained highly effective antibacterial properties. In conclusion, the results indicated that $\mathrm{G}-$ and $\mathrm{G}+$ bacteria were efficiently and remarkably inhibited. antibacterial agents had a slight or no dissolution. The
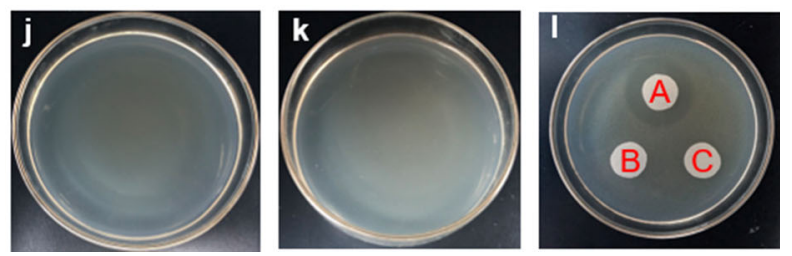

1, 5, 10, and $30 \mathrm{~min}$. Inhibition zone experiments on the samples: $\mathbf{f}$ E. coli and $\mathbf{I}$ S. aureus. Note: A, B, and C are untreated cotton-added antibiotics and untreated cotton used as the positive and negative controls and antibacterial cotton, respectively
Table 1 Biocidal efficacy against microorganisms

\begin{tabular}{|c|c|c|c|}
\hline \multirow[t]{3}{*}{ Sample } & \multirow[t]{3}{*}{ Contact time (min) } & \multicolumn{2}{|c|}{ Bactericidal ratio } \\
\hline & & E. $\operatorname{coli}^{\mathrm{a}}$ & S. aureus $^{\mathrm{b}}$ \\
\hline & & $\%$ reduction & $\%$ reduction \\
\hline Untreated cotton & 30 & - & - \\
\hline \multirow[t]{4}{*}{ Cl-AOBYID-cotton $\left(0.38 \% \pm 0.01 \mathrm{Cl}^{+}\right)$} & 1 & 97.83 & 94.83 \\
\hline & 5 & 99.28 & 100 \\
\hline & 10 & 100 & 100 \\
\hline & & 100 & 100 \\
\hline
\end{tabular}

${ }^{\mathrm{a}}$ Inoculum concentration was $5.56 \times 10^{8} \mathrm{CFU} /$ sample

${ }^{\mathrm{b}}$ Inoculum concentration was $2.76 \times 10^{8} \mathrm{CFU/}$ sample 
a

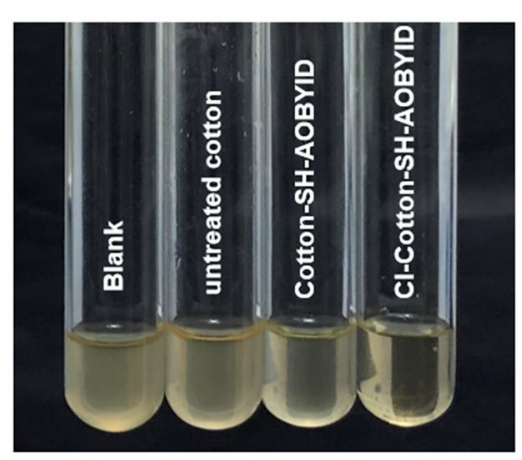

b

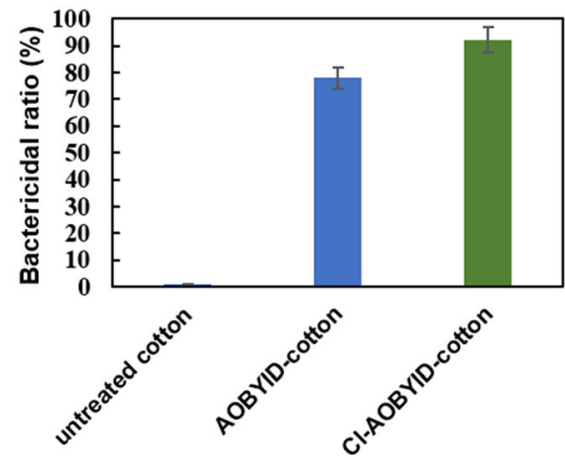

Fig. 4 Antibacterial activity of the treated cotton fabrics a images of the E. coli and $S$. aureus solutions that were cocultured with the treated cotton fabrics after $24 \mathrm{~h}$.

The antibacterial activity of the treated cotton fabric with prolonged contact was further explored. The AOBYID-treated cotton fabrics displayed antibacterial activities likely due to the presence of the Schiff base, whereas the chlorinated cotton fabrics demonstrated strong antibacterial activities likely due to the antimicrobial broad spectrum of $N$-halamine. Figure $4 \mathrm{a}$ shows the image of bacterial suspensions that were cultured with samples. The suspensions of the blank and untreated cotton samples were turbid during the $24 \mathrm{~h}$ culture, whereas those of cotton-SHAOBYID and Cl-cotton-SH-AOBYID were relatively clear. The bactericidal ratio of cotton-SH-AOBYID for E. coli and S. aureus were $78 \%$ and $89 \%$, respectively, possibly due to the presence of the Schiff base structure. However, the bactericidal ratios of $\mathrm{Cl}$ cotton-SH-AOBYID for E. coli and S. aureus were $92 \%$ and $95 \%$, respectively, probably because the chlorinated sample had increased antibacterial activity
S. aureus

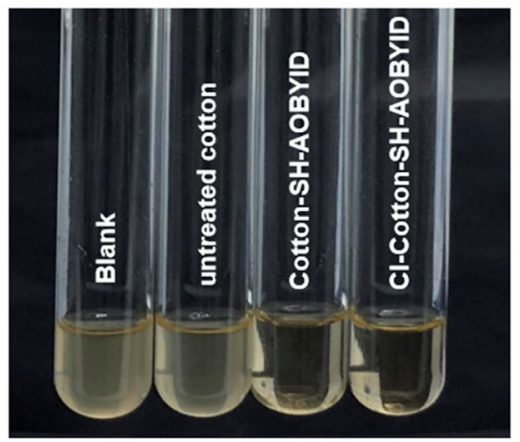

S. aureus

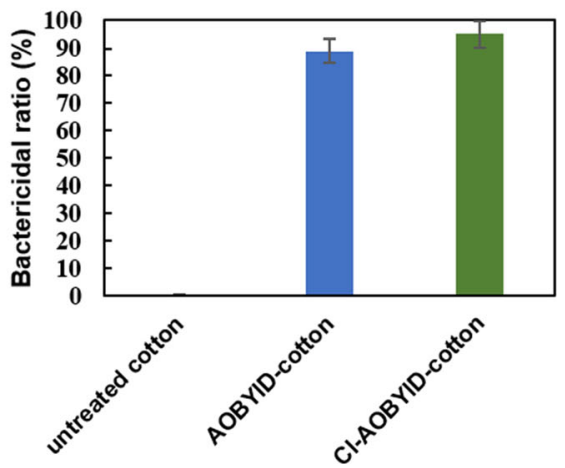

b Bactericidal ratio of the treated cotton fabrics with $S$. aureus and $E$. coli (The error bar indicates the standard deviation)

(Fig. 4b). These results were consistent with previous reports (Table 1).

Storage stability and UV stability

The stability of Cl-cotton-SH-AOBYID was evaluated, and the results are presented in Fig. 5a. Although the $\mathrm{Cl}+$ content of the tested samples gradually decreased with the increase in storage time, nearly $79 \%$ of the initial chlorine loading still remained after 30 days of storage (oxidative chlorine decreased from 0.38 to $0.30 \%$ ). The stability combined with the antibacterial efficacy result were sufficient to support its application. Chlorine loss could have been caused by the hydrolysis of $\mathrm{N}-\mathrm{Cl}$ bonds (Zhang et al. 2019). Notably, after 30 days of storage, rechlorination of the sample could restore the original content of chlorine oxide.

The UVA light stability of the chlorinated cotton fabric was further tested. Figure $5 \mathrm{~b}$ shows that active 

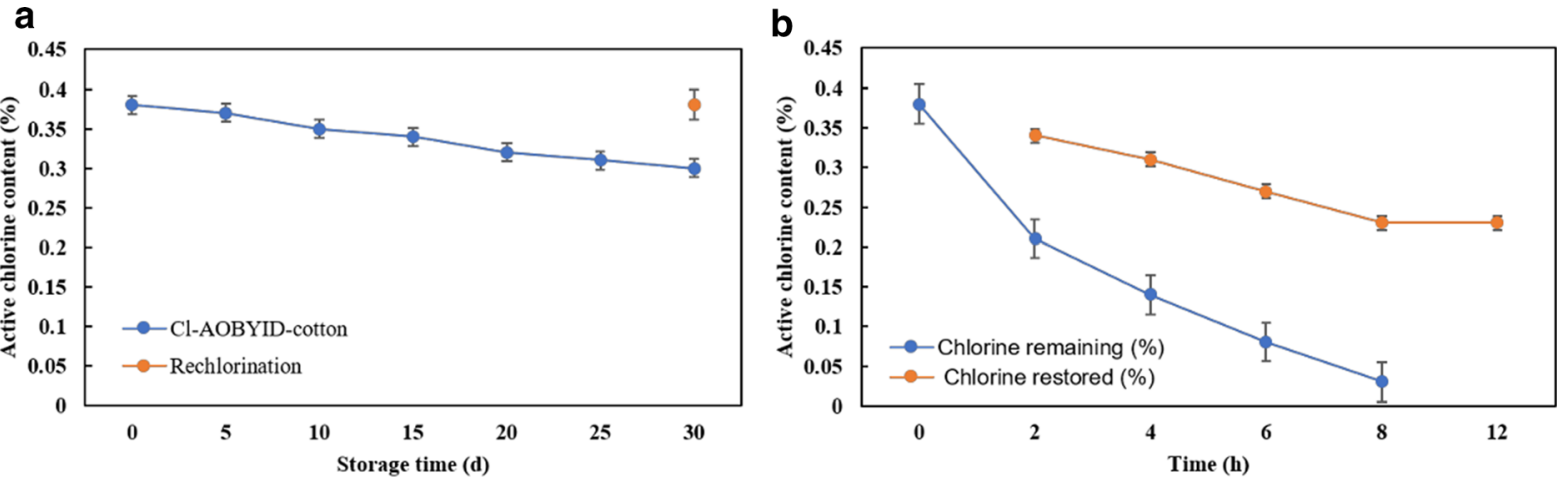

Fig. 5 a Storage and $\mathbf{b}$ UV stability of active chlorine on the treated fabric (The error bar indicates the standard deviation)

Table 2 Washing durability of the antibacterial finish cotton fabric

\begin{tabular}{|c|c|c|}
\hline Washing cycles & $\begin{array}{l}\text { E. } \text { coli }^{\mathrm{a}} \\
\text { Bacterial reduction }(\%)\end{array}$ & $\begin{array}{l}\text { S. } \text { aureus }^{\mathrm{b}} \\
\text { Bacterial reduction }(\%)\end{array}$ \\
\hline 0 & 91.35 & 94.80 \\
\hline 5 & 85.58 & 87.35 \\
\hline 10 & 78.71 & 80.70 \\
\hline
\end{tabular}

${ }^{a}$ Inoculum concentration was $7.67 \times 10^{8} \mathrm{CFU} /$ sample

${ }^{\mathrm{b}}$ Inoculum concentration was $4.82 \times 10^{8} \mathrm{CFU} / \mathrm{sample}$

chlorine on the cotton fabrics decreases with the increase in irradiation time. The fastest increase occurred in the first hour of irradiation, and approximately $45 \%$ of chlorine oxide remained on the fabric. After $12 \mathrm{~h}$ of UV irradiation, active chlorine was almost completely lost. However, 60\% of chlorine could be regenerated through contact with the diluted sodium hypochlorite solution. Therefore, the results showed that Cl-cotton-SH-AOBYID demonstrated good rechargeability.

Washing durability

According to the standard of AATCC 61-2006, the antibacterial finish fabrics were washed repeatedly, and then the antibacterial durability was evaluated. Table 2 shows the test results. After 5 and 10 washing cycles, the antibacterial fabric still maintained an antibacterial rate of 85.58 and $78.71 \%$ against E. coli, $87.35 \%$ and $80.70 \%$ against S. aureus, respectively. Consequently, the antibacterial cotton fabrics still maintained a high antibacterial rate after repeated washing.

\section{Conclusions}

${ }^{1} \mathrm{HMR}, \mathrm{FT}-\mathrm{IR}$, and Raman results indicated that the Hein-Schiff base compounds were successfully synthesized and grafted onto the cotton fibers. Antibacterial activity test revealed that the prepared antibacterial cotton fabric had high efficiency and good antibacterial activity against $S$. aureus and E. coli, and its antibacterial performance was slightly better against $S$. aureus. The presence of the Schiff base structure in the finishing agent enhanced the antibacterial activity, and the introduction of halogenated amine bonds in the grafted cotton fabric also significantly improved the antibacterial properties of the treated cotton fabric. The stability test results of storage and UV presented that the presence of aromatic rings effectively enhanced the stability, and the treated fabric exhibited good rechargeability. The antibacterial fabric still retained high antibacterial performance after repeated washing.

Acknowledgments This work was supported by the Hunan Province Key R \& D Projects(2016GK2020) and the Opening Project of Hunan Engineering Laboratory for Preparation Technology of Polyvinyl Alcohol Fiber (HGY201607). 


\section{References}

Cai Q, Yang S, Zhang C, Li Z, Li X, Shen Z, Zhu W (2018) Facile and versatile modification of cotton fibers for persistent antibacterial activity and enhanced hygroscopicity. ACS Appl Mater Interfaces 10(44):38506-38516

Chen W, Zhu Y, Zhang Z, Gao Y, Liu W, Borjihan Q, Qu H, Zhang Y, Zhang Y, Wang Y-J, Zhang L, Dong A (2020) Engineering a multifunctional $\mathrm{N}$-halamine-based antibacterial hydrogel using a super-convenient strategy for infected skin defect therapy. Chem Eng J 379:122238

Fan J, Bao B, Wang Z, Xu R, Wang W, Yu D (2019) High tristimulus response photochromic cotton fabrics based on spiropyran dye by thiol-ene click chemistry. Cellulose 27(1):493-510

Fei Z, Liu B, Zhu M, Wang W, Yu D (2018) Antibacterial finishing of cotton fabrics based on thiol-maleimide click chemistry. Cellulose 25(5):3179-3188

Gan D, Xing W, Jiang L, Fang J, Zhao C, Ren F, Fang L, Wang K, Lu X (2019) Plant-inspired adhesive and tough hydrogel based on Ag-Lignin nanoparticles-triggered dynamic redox catechol chemistry. Nat Commun 10(1):1487

Hassan MA, Omer AM, Abbas E, Baset WMA, Tamer TM (2018) Preparation, physicochemical characterization and antimicrobial activities of novel two phenolic chitosan Schiff base derivatives. Sci Rep 8(1): 11416

Herwig G, Dove AP (2019) Synthesis of rapidly surface eroding polyorthoesters and polyacetals using thiol-ene click chemistry. ACS Macro Lett 8(10):1268-1274

Küçük M, Öveçoğlu ML (2019) Fabrication of SiO2-ZnO NP/ $\mathrm{ZnO}$ NR hybrid coated cotton fabrics: the effect of $\mathrm{ZnO}$ NR growth time on structural and UV protection characteristics. Cellulose 27(3):1773-1793

Liu B, Deng X, Xie Z, Cheng Z, Yang P, Lin J (2017) Thiol-ene click reaction as a facile and general approach for surface functionalization of colloidal nanocrystals. Adv Mater 29(36): 1604878

Liu J, Dong C, Wei D, Zhang Z, Xie W, Li Q, Lu Z (2019) Multifunctional antibacterial and hydrophobic cotton fabrics treated with cyclic polysiloxane quaternary ammonium salt. Fibers Polym 20(7):1368-1374

Ma K, Liu Y, Xie Z, Li R, Jiang Z, Ren X, Huang T-S (2013) Synthesis of novel N-halamine epoxide based on cyanuric acid and its application for antimicrobial finishing. Ind Eng Chem Res 52(22):7413-7418

Ma W, Li L, Liu Y, Sun Y, Kim IS, Ren X (2019a) Tailored assembly of vinylbenzyl N-halamine with end-activated $\mathrm{ZnO}$ to form hybrid nanoparticles for quick antibacterial response and enhanced UV stability. J Alloy Compd 797:692-701
Ma W, Li L, Ren X, Huang T-S (2019b) Highly effective antibacterial polycaprolactone fibrous membranes bonded with N-Halamine/ZnO hybrids. Surf Coat Technol 379:125021

Naeimi H, Sharghi H, Salimi F, Rabiei K (2008) Facile and efficient method for preparation of schiff bases catalyzed by $\mathrm{P} 2 \mathrm{O} 5 / \mathrm{SiO} 2$ under free solvent conditions. Heteroat Chem 19(1):43-47

Qiao M, Ren T, Huang T-S, Weese J, Liu Y, Ren X, Farag R (2017) N-Halamine modified thermoplastic polyurethane with rechargeable antimicrobial function for food contact surface. RSC Adv 7(3):1233-1240

Rattanaphani S, Chairat M, Bremner JB, Rattanaphani V (2007) An adsorption and thermodynamic study of lac dyeing on cotton pretreated with chitosan. Dyes Pigm 72(1):88-96

Ren X, Kou L, Liang J, Worley SD, Tzou Y-M, Huang TS (2008) Antimicrobial efficacy and light stability of N-halamine siloxanes bound to cotton. Cellulose 15(4):593-598

Rohani Shirvan, A., S. Kordjazi and A. Bashari (2019) Environmentally friendly finishing of cotton fabric via star-like silver micro/nano particles synthesized with Neem/Salep. J Nat Fibers 1-9

Rong L, Liu H, Wang B, Mao Z, Xu H, Zhang L, Zhong Y, Feng X, Sui X (2019) Durable antibacterial and hydrophobic cotton fabrics utilizing enamine bonds. Carbohyd Polym 211:173-180

Sandstrom A, Sun G, Morshed M (2007) Biocidal aramide fabrics for emergency responders: formation and properties of aramide halamine. Text Res J 77(8):591-596

Xu L, Wang W, Yu D (2017) Durable flame retardant finishing of cotton fabrics with halogen-free organophosphonate by UV photoinitiated thiol-ene click chemistry. Carbohydr Polym 172:275-283

Yousif E, Ahmed DS, El-Hiti GA, Alotaibi MH, Hashim H, Hameed AS, Ahmed A (2018) Fabrication of novel balllike polystyrene films containing schiff base microspheres as photostabilizers. Polymers (Basel) 10(11):1185

Zhang W, Dai X, Zhou J (2018) Properties of cotton fabric modified with a chitosan quaternary ammonium salt nanoparticle. Fibres Text Eastern Eur 26:116-121

Zhang S, Kai C, Liu B, Zhang S, Wei W, Xu X, Zhou Z (2019) Preparation, characterization and antibacterial properties of cellulose membrane containing N-halamine. Cellulose 26(9):5621-5633

Publisher's Note Springer Nature remains neutral with regard to jurisdictional claims in published maps and institutional affiliations. 